part of the oesophagus in these cases is completely freed by dividing all the fibrous mediastinal attachments which have resulted from peri-oesophagitis, the normal part of the tube is nearly always sufficiently elastic to allow the hernia to be reduced without undue tension. I am sure that placing the anchoring stitches from the insertion of the phreno-oesophageal ligament to the under surface of the hiatal muscle as described and tightening them all together is an important factor in making an oesophagus which is apparently short lengthen sufficiently for good repair of the hernia. Bringing the oesophagus through the anterior part of the hiatus is also important, i.e., always placing the hiatal stitches behind the oesophagus, never at the sides or in front, because the plane of the hiatus is tilted forwards very little from the vertical so that the most anterior part is also the highest.

There is another condition which has been called " short oesophagus." Those who believe that only the part of the tube lined by squamous epithelium can rightly be called oesophagus consider that a state of short oesophagus exists when columnar epithelium extends further than usual up the tube, whether the tube itself is shortened or not. In the previous paper I have explained why I do not recognize reduction in the length of the part of the tube lined by squamous epithelium as a type of short oesophagus.

\section{SUMMARY}

The insertion of the phreno-oesophageal ligament into the oesophagus is at a level about $2 \mathrm{~cm}$. above the oesophago-gastric junction.

The fixation of this insertion to the lower edge of the hiatus is an essential step in the repair of a hiatal hernia. A method of doing this is described.

Neither the wall of the oesophagus nor of the stomach should be included in any stitches used in the repair.

Repair by the method described makes an oesophagus too short to allow restoration of the stomach to the abdomen a rare finding.

\section{REFERENCES}

Allison, P. R. (1951). Surg. Gynec. Obstet., 82, 419.

Burke, J. B. (1959). Lancet, 2, 787.

Clarebrough, J. K., and Connell, J. L. (1959). Aust. N.Z. J. Surg., $29,21$.

Groves, L. K., Martinez, W. V., and Effler, D. B. (1959). J. thorac. Surg., 38, 537.

Hagarty, G. (1959). Med. J. Aust., 2, 515.

Harrington, S. W. (1955). Surg. Gynec. Obstet., 100, 277.

Laimer, E. (1883). Beitrag Zur Anatomie Des Oesophagus, Med. Jahrbücher (Wien), p. 333. (Quoted by Hagarty, S.)

Lerche, W. (1950). The Esophagus and Pharynx in Action, p. 65. Thomas, Springfield, Illinois.

Marchand, P. (1959). J. thorac. Surg., 37, 81.

Peters, P. M. (1955). Thorax, 10, 27.

Sweet, R. H. (1948). New Engl. J. Med., $238,649$.

Tanner, N. C. (1955). Lancet, 2, 1050.

\title{
THE TREATMENT OF FIBROUS STRICTURE OF THE OESOPHAGUS ASSOCIATED WITH HIATAL HERNIA
}

When a patient with a sliding hiatal hernia develops dysphagia, this symptom may be due to inflammatory hyperaemia and oedema in the oesophagus, to oesophageal muscular incoordination or spasm, or to the laying down circumferentially of dense, contracted fibrous tissue in the mucosa, the mucosa and submucosa, or all layers of the oesophageal wall. These changes can occur separately or together. In this paper a stricture is regarded as fibrous when there is convincing evidence at oesophagoscopy and at operation of the presence of circumferential fibrosis in the oesophagus sufficient in itself to cause significant oesophageal obstruction whether the other factors are also present or not. These fibrous strictures complicating sliding hiatal hernias are difficult to treat satisfactorily, and an extraordinarily wide range of procedures has been suggested-a sure sign that the best solution to the problem has yet to be found.

Most authors favour some sort of resection (Allison, 1951 ; Allison, Wooler, and Gunning, 1957 ; Barrett, 1952, 1957 ; Belsey, 1953 ; Ellis, 1956 ; Ellis, Andersen, and Clagett, 1956 ; Lindskog and Kline, 1957 ; MacLean and Wangensteen, 1956 ; Mustard, 1957 ; Sweet, Robbins, Gephart, and Wilkins, 1954 ; Tanner, 1955 ; Wooler, 1956), but few agree on what is to be removed or how best to reconstitute the alimentary canal afterwards. Many variations of resection of oesophagus, or stomach, or both, with all sorts of anastomoses which may or may not involve the jejunum or colon, have been advocated. Sometimes no stomach, sometimes the upper part of it, sometimes the lower part, sometimes the upper and lower parts leaving a portion of the greater curvature in 
the middle, is removed. No one as yet seems to remove all the stomach for this condition. The results are always said to be satisfactory. I do not believe it. If the results really satisfied the surgeons and patients, ingenious new pieces of alimentary mutilation would not continue to be devised for this condition.

Some reports favour more conservative measures. Benedict and Nardi (1957) prefer no operation at all. They advise dilatations with bougie olives guided along a swallowed thread. Presumably these dilatations are repeated for the rest of the patients' lives. They resort to surgery if this treatment fails or severe haemorrhage occurs, and then occasionally do an oesophagoplasty; but usually advise resections. Several authors advise repair of the hernia, if possible, followed by post-operative endoscopic dilatation of the stricture (Holinger, Johnston, Potts, and daCunha, 1954), or by dilatation during operation by bougies passed by the anaesthetist and guided by the surgeon (Byrnes, 1958), or by retrograde dilatation via the stomach at the time of operation (Burke, 1959 ; Groves, Martinez, and Effler, 1959). Cross, Smith, and Kay (1959) stated that " repair of the associated hiatal hernia, rather than one of the more complicated procedures," was their treatment of choice, but they did not give any details about what they did to the stricture. Berman and Berman (1959) also think that the hernia should be repaired, followed by post-operative endoscopic dilatations, but they added vagotomy, restoration of the cardio-oesophageal angle by oesophagogastropexy, and pyloroplasty with excision of the anterior third of the pyloric sphincter to the operation. They cannot have much faith in their method of repair of the hernia.

This summary indicates that a surgeon, faced with the problem of a patient with hiatal hernia and oesophageal stricture and seeking guidance, will turn to the literature in vain. It will only confuse him. I have found it best to ignore the literature and work out the management of my patients in my own way. This paper is based on 14 consecutive patients, all with tough fibrous strictures and persistent dysphagia. Patients with mild or intermittent dysphagia due to superficial oesophagitis are excluded.

The experience of these patients has led to the conclusion that resections are seldom, if ever, needed and that good results can be obtained by repair of the hernia, dilatation of the stricture to the point of internal splitting at the time of operation, and post-operative dilatation by selfbouginage with Hurst's mercury bougies for a limited period when necessary. The evidence presented here is purely clinical. Since no resection was done pathological specimens were not available for study.

\section{Pre-operative Assessment}

The history of peptic oesophagitis, often for a long period, followed by dysphagia, sometimes rapidly developing and sometimes present for many years or from infancy, and perhaps associated with haemorrhage and anaemia, is very characteristic in all its variations and should lead to the correct provisional diagnosis. Barium studies must be adequate, with full outlining of the stomach and duodenum. If no barium or insufficient barium passes the stricture at the first examination, it must be repeated after dilatation of the stricture through the oesophagoscope. The radiological examination is not complete until the hiatal hernia is properly shown and until it is proved with reasonable certainty that there is no chronic ulcer in the stomach or duodenum and no carcinoma in the stomach. If these conditions are found, gastric resection is necessary, as it would be if there were no associated hernia and oesophageal stricture. They are the only indications for resection. The stricture in the oesophagus is not an indication for resection of oesophagus or stomach.

At oesophagoscopy the possibility of oesophageal carcinoma is excluded by inspection and biopsy. Solid pieces of food which have failed to pass the stricture may be present and need to be removed. The stricture is dilated with bougies as far as is possible with safety, and, if the stricture splits at this stage, there will be less dilatation to do at operation. In these cases one commonly feels a second, lesser resistance to the passage of the bougies, usually 2 or $3 \mathrm{~cm}$. below the stricture. This is due to muscular spasm at the oesophagogastric junction.

This spasm also makes it very difficult for radiologists to fill the oesophagus below the stricture. Often they fail to do so. Then in the films the stricture looks as if it extends down to the oesophago-gastric junction, and even when it is only a narrow ring at the top of the length of apparent narrowing, it seems to be 2 or $3 \mathrm{~cm}$. long. The stricture never extends down as far as the oesophago-gastric junction (Hayward, 1961a). It may, however, extend upwards (ascending oesophagitis) making a long length of narrowing, or be short but situated as high as the aortic arch with a long length of unstrictured oesophagus below instead of the usual 2 or $3 \mathrm{~cm}$. Long strictures are usually relatively easily dilatable with bougies as far as their lower ends. The bougie may not 
pass the extreme lower end, because the densest fibrous tissue is usually limited to the lower end of the stricture. The rest is largely due to hypertrophy and spasm of the muscular wall of the oesophagus. Thus, in my experience, however long they may look, tough fibrous strictures in these cases are usually relatively short and sometimes annular.

Having established the nature and position of the stricture and excluded associated gastric or duodenal disease, the patient is prepared for operation. Blood transfusion to correct anaemia may be required and a period on high-protein, high-vitamin fluids and vitaminized food may be necessary. The oesophagoscopic dilatation already done usually relieves the dysphagia for at least 10 to 14 days and enables the patient to ingest enough fluid and soft food to be brought into reasonable condition for surgery. If dilatation has failed and dysphagia is complete, intravenous fluids are necessary. The patient should have the operation as soon as he is fit. He may feel so much better after the endoscopic dilatation that he wishes to defer it. He should be advised against this and warned that his dysphagia will surely recur as severely as ever, probably in a very short time.

\section{The Operation}

As many fibrous strictures are associated with peri-oesophagitis which has produced dense mediastinal adhesions, the approach must give direct access to the stricture to enable this part of the oesophagus to be dissected free. The chest must, therefore, be opened; a purely abdominal approach in this type of case is inadequate. If there is chronic peptic ulceration in the stomach or duodenum requiring gastrectomy, a thoracoabdominal incision is best. Otherwise an ordinary postero-lateral thoracotomy is used, usually on the left side, but occasionally on the right side for a a very high stricture which would require difficult dissection under the aortic arch if approached from the left. The hernia can be satisfactorily repaired through a right thoracotomy.

Surgical access having been obtained, the operation proceeds in a series of clearly defined steps.

Freeing the Oesophagus.-This may be easy, but is more often tedious and difficult. It should be done in the plane outside the vagi and the paraoesophageal lymph glands which in these cases are enlarged, so that these structures remain intact on the oesophageal wall. The inflammation round the oesophagus may have obliterated the tissue planes. Then it is best to define the correct plane round the normal oesophagus above or below the stricture and work down or up from this. Much of the dissection will need to be sharp. As the inflammatory tissue is very vascular, many small and often very short arteries entering it from the descending aorta will be divided and require careful ligation. The opposite pleura will probably be opened: with controlled respiration anaesthesia this does not matter. I have not drained the opposite pleural cavity when it has been opened and have not needed to aspirate it post-operatively. When the strictured area is quite free, the dissection continues easily downwards in the same plane over the lower oesophagus and thoracic stomach with its associated peritoneal sac to the muscular margins of the hiatus in the same way as is done for repair of a hiatal hernia without a stricture.

The whole lower oesophagus and hernia are now lying quite free in the mediastinum, and the strictured part of the oesophagus looks and feels very thick, but its longitudinal muscle coat is clearly visible on the outside and appears intact in spite of the violence of the inflammatory process around it. The abnormal thickness of the oesophageal wall may continue up from the stricture for a few centimetres. The thickened part above the stricture does not feel quite so firm and seems to be due largely to hypertrophy of the muscular coat. Presumably this is a normal response to the presence of the obstruction in the lumen, as would be expected in any tube with a muscular coat.

Splitting the Stricture from Within.-At this stage the anaesthetist is asked to pass Hurst mercury bougies, beginning with No. 22 (English). The surgeon palpates the tip of the bougie through the oesophageal wall as it approaches the stricture and with one hand directs it into the stricture while the other hand encircles the oesophagus lightly to judge the progress of the bougie and help guide it through. With this guidance of the tip, the anaesthetist may safely use all the force the bougie will take short of kinking or curling up in the throat. After being held up for a few seconds the bougie may suddenly go through because it has split the constricting fibrous tissue in the mucosa and submucosa, probably together with any epithelium that may be over it. It is surprising how often this will occur with this method even when a much smaller bougie could not be passed safely at the pre-operative oesophagoscopy. Once the first bougie has passed one can usually proceed easily through the series up to No. 30 (English). The dilatation is not adequate until No. 30 will pass freely without being gripped, 
i.e., until all the constricting fibrous tissue in the mucosa and submucosa has been split. The stricture, which was easily palpable from the outside before, is now impalpable, though the palpable muscular hypertrophy, of course, remains.

If the bougies fail to pass the stricture, retrograde dilatation is necessary. For low strictures this is done through a small gastrotomy in the thoracic part of the stomach after exposing it by dividing the phreno-oesophageal ligament and sac just above the hiatus. For high strictures at the level of the aortic arch it is done through a small longitudinal oesophagotomy in the normally patent oesophagus just below the stricture.

The stricture is first palpated with the finger up the oesophagus. Sometimes the finger succeeds in splitting it. If not, instruments must be used. After trying various implements, I have come to the conclusion that the best are Hegar's dilators up to the size of Brock's expanding aortic valve dilator followed by this instrument opened cautiously but with considerable force until splitting suddenly occurs. This has happened before it was fully open. I have not needed to open it fully and have hesitated to do so for fear of splitting the muscle. As with the bougies, these retrograde instruments are guided very carefully through the stricture with the hand encircling it on the outside. After the stricture has split the proof that dilatation is adequate is given by retrograde palpation by the finger and the passage of the No. 30 Hurst bougie from above. Neither should be gripped. A little bleeding into the oesophageal lumen results from the splitting, but, from my experience, it can be ignored.

After dilatation of the stricture the anaesthetist passes a Ryle's tube into the stomach and brings it out through the nose, where it is fixed with strapping at the correct length. This is for postoperative gastric aspirations until intestinal peristalsis is resumed. If retrograde dilatation has been necessary the gastrotomy or oesophagotomy is closed.

Repairing the Hernia.-The details are as for repair of any sliding hiatal hernia. If the stricture is to disappear post-operatively, the repair must completely stop all oesophageal reflux. This method of dealing with these cases will assuredly fail if the repair of the hernia is imperfect. I believe that the repair is imperfect if the attachment of the phreno-oesophageal ligament to the oesophagus is not properly fixed below the hiatus (Hayward, 1961b). If the oesophagus is fully freed, as described above, there will rarely be any difficulty in achieving this. It is uncommon for the oesophagus to be too short. Though I have never found it so, my colleague, Mr. Ian McConchie, did on one occasion when he was performing this operation on a boy of 15 with a congenital hernia and ascending oesophagitis and fibrous stricture. He managed the reduction by transposing the oesophagus to an opening in the higher, membranous portion of the left diaphragm, and, in the ensuing months, the boy's stricture has disappeared. A newly created hiatus can, therefore, be adequate.

\section{Post-operative Care}

After this operation the oesophageal lining is left split by the dilatation as well as eroded by any ulceration that was present pre-operatively. As the reflux has been stopped this will heal, but, until the epithelialization of denuded areas is complete, more fibrous tissue will form and may contract. After epithelialization is complete any fibrous tissue present underneath will gradually thin out and cease to constrict.

In addition to routine post-operative treatment these patients need watching for the least sign of a return of dysphagia. The cessation of reflux makes all medical measures such as dietary restrictions, alkalis, antispasmodics, and sleeping propped up unnecessary. I put them on ordinary solid food as soon as the alimentary canal will take it, usually early in the second week. Good boluses of solid food are useful dilators and, by being held up in their passage down the oesophagus, immediately indicate any return of the narrowing. This happens in more than half the cases, and, when it does, the patient must start self-bouginage at once, aiming at size No. 26 Hurst. If this size will go through there will be no trouble with a full normal diet.

Self-bouginage may be required daily at first, but, as soon as possible, the intervals are lengthened. The patient goes home with his No. 26 (English) bougie, and when he can omit passing it for a week without return of dysphagia and without it being gripped when he swallows it after this interval, he can discard it. This normally happens within a few weeks or, at most, a few months. The aim is to keep the stricture open until all ulceration has healed. Then it will stay open by itself. Patients will, as a rule, face up to self-bouginage when it is explained that it is only to go on for a short time, not the rest of their lives. On occasion I have had difficulty in persuading them to relinquish the bougie. They are so afraid of a return of the awful misery of dysphagia that it may be hard to convince them that it really will not recur. 
If self-bouginage has been started too late and No. 26 will not pass the stricture, oesophagoscopic dilatation to size No. 28 , which goes through the Negus full lumen oesophagoscope, is necessary. Then the patient will be able to swallow the No. 26, starting the next day. Lubricating it with xylocaine jelly helps while the throat is still sore after oesophagoscopy.

\section{Clinical Material and Comments}

The 14 cases on which this report is based were consecutive in the author's practice during the past four years, but must not be taken to represent the true incidence of the condition or the relative frequency of its variations. As the practice includes very little paediatric work, no infants or children are included. Also, being a purely thoracic surgical practice, cases with associated chronic gastric or duodenal ulceration tend only to be referred from the general surgeon if he encounters thoracic trouble with which he needs help. The incidence of this ulceration in this series (three out of 14) may therefore be too low.

The patient's ages varied from 17 to 84 and averaged 53 years.

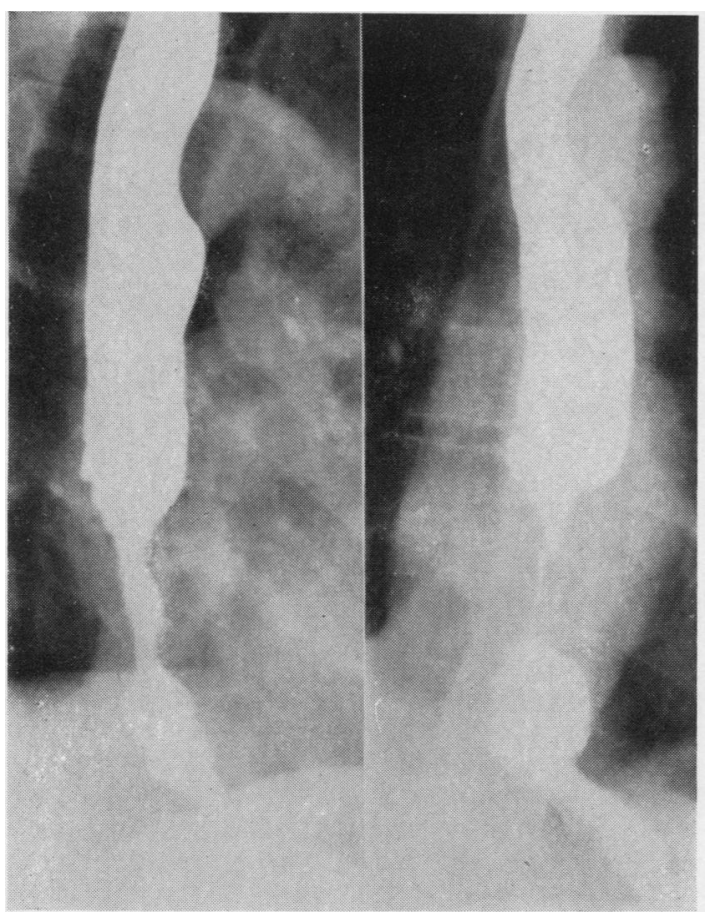

FIG. 1.-Radiograph of a low, short stricture in a man aged 63. Two views at the same barium examination. In the film on the left the radiologist has succeeded in filling the oesophagus below the stricture. The second narrowing is due to muscular spasm at the oesophago-gastric junction.

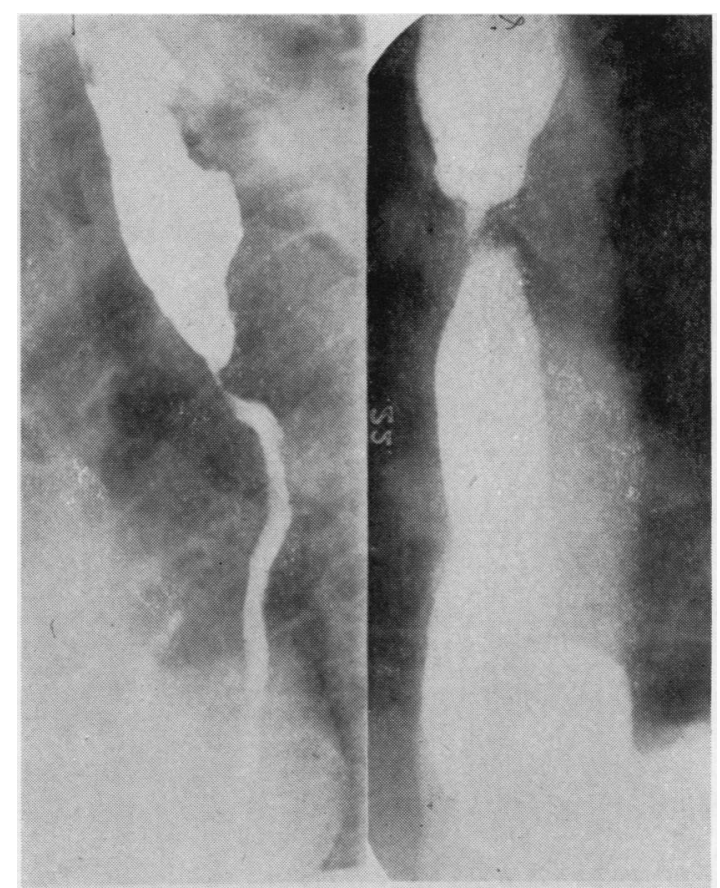

FIG. 2.-Radiograph of a high, short stricture in a man aged 28. Two views at the same barium examination. Often, radiologists only produce films like the one on the left, which leave the pathology in doubt. The one on the right shows the full picture of hiatal hernia and stricture.

There were eight men and six women. In two the history of dysphagia began at the time of weaning, strongly suggesting that the stricture was secondary to a congenital hiatal hernia. In the remaining 12 the digestive troubles and dysphagia did not begin till well into adult life and there was little doubt that they were all due to acquired hiatal hernias.

The strictures fell into three fairly well defined groups: 10 low, short strictures (Figs. 1, 4, and 5), three high, short strictures (Fig. 2), and one long stricture (Fig. 3). In all of them the maximum narrowing was localized to a portion of the oesophagus not more than $2 \mathrm{~cm}$. long and usually less, and the narrowing seemed to be due to fibrosis internal to the muscle coat, i.e., in the mucosa, or submucosa, or both.

In 12 it was impossible to be more specific than this because they were not seen from inside except through the oesophagoscope. In the remaining two the stricture was opened longitudinally for an oesophagoplasty, and in one of these the very narrow part at the lower end was definitely due to mucosal and submucosal fibrosis and was barely $1 \mathrm{~cm}$. long. The other $6 \mathrm{~cm}$. of lesser narrowing 


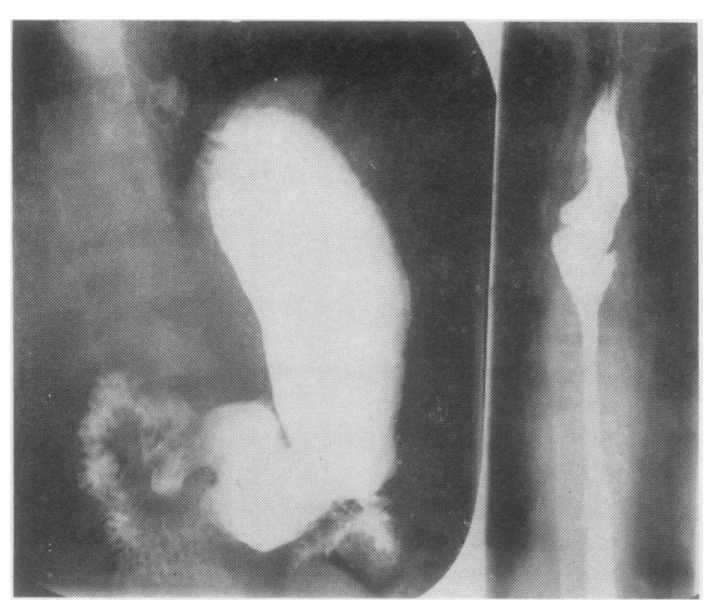

FIG. 3.-_Radiograph of a long stricture in a lad aged 17, secondary to a congenital hiatal hernia shown on the left. At this examination the radiologist took no picture showing the hernia and stricture on the one film.

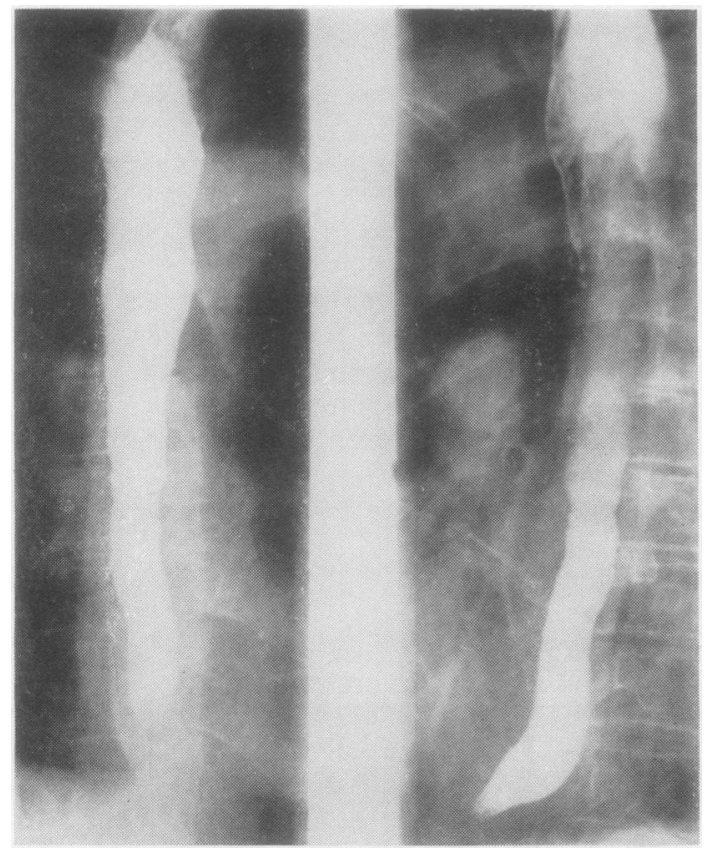

FIG. 4.-Radiograph of a low stricture in a man aged 64 . This stricture was associated with muscular hypertrophy of the lower oesophagus, sufficient to be visible in the films as a line outside the column cf barium. No barium passed the stricture, so the hernia is not shown, but a small one was found at operation.

above, found at oesophagoscopy, was apparently due to hypertrophy of the muscle coat. In the lower centimetre which was incised for the oesophagoplasty the muscle was seen to be $1 \mathrm{~cm}$. thick (Fig. 4). In several other cases the oesophageal muscle for a few centimetres above the stricture seemed hypertrophied, but, as it was only inspected $\frac{\bar{\sigma}}{\bar{D}}$. and palpated from the outside in these cases, it was $\frac{\text { Th }}{\vec{D}}$

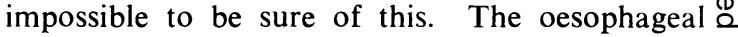
wall of the second case in which oesophagoplasty was done seemed very fibrous in all layers at the $\vec{\circ}$ site of the stricture, which was a high short one. This: was also the only case in which the longitudinal $\vec{\omega}$ muscle coat did not appear normal from the $\stackrel{\Omega}{\rightarrow}$ outside, but seemed fibrosed. Whether this stricture $\vec{x}$ would have been relieved by internal splitting if $\overrightarrow{\vec{\sigma}}$ I had been able to do this satisfactorily is not known.

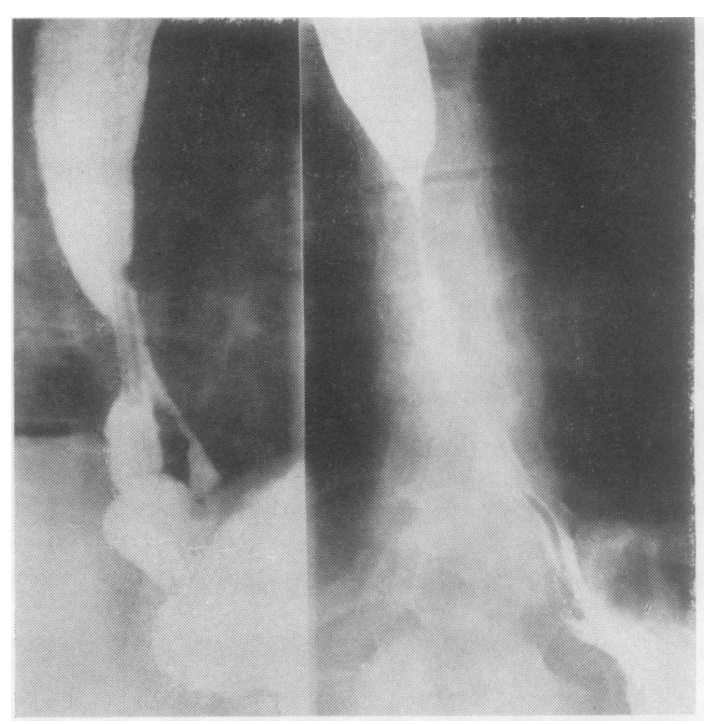

FIG. 5.-Radiograph of a low, short stricture in a woman aged 32 . A penetrating ulcer has leaked into the mediastinum (left-hand film). The "waist" below the stricture at the oesophago-gastric junction is well seen. At this stage the stricture had been dilated sufficiently to allow the passage of a Ryle's tube for a continuous intragastric drip to supplement oral feedings. The "leaking ulcer" could have been due to a bougie, but all the bougies $\frac{7}{0}$ seemed to pass normally and there was no pain after the oesophagoscopy, only delight at the improvement in the dysphagia. $N$ The film on the right was taken before oesophagoscopy. Insuffi. $O$ cient barium passed the stricture to distend the lower oesophagus $\mathrm{N}$ and stomach at this examination.

In a small series of 14 cases it is unlikely that all the possible variants would be encountered. There may be some with so much muscle fibrosis that internal splitting is not feasible, but I think $\stackrel{0}{+}$ it can be accepted that, in most cases of stricture due to peptic oesophagitis, the muscle coat is remarkably healthy and internal splitting of the stricture is effective and safe.

Hypertrophy of the muscle coat of the oesophagus in association with these strictures has been noted by Barrett (1957). Groves et al. (1959) also observed it, even in some cases of peptic 
oesophagitis without stricture; they stated that they often did a myotomy for it. To damage muscle which is reacting normally to the presence of obstruction seems unnecessary and is useless, as they admitted, for tough fibrous strictures where the contracting fibrous tissue is in the mucosa and submucosa.

In the group of 10 low, short strictures there were two in which the stricture consisted of a tough, fibrous, annular diaphragm with a small, central hole, situated about $2 \mathrm{~cm}$. above the oesophago-gastric junction. Similar strictures have previously been described as lower oesophageal ring, lower oesophageal web and congenital oesophageal ring. MacMahon, Schatzki, and Gary (1958) noticed that these rings were always above the diaphragm and always associated with a hiatal hernia. They said that they always seemed to be at the junction of oesophagus and stomach, by which they meant at the junction of squamous and columnar epithelium, but this is above the oesophago-gastric junction as defined by me (Hayward, 1961a).

Allison et al. (1957) and Smith and Bradshaw (1959) advised submucous resection of them. Bugden (1957) described triradiate incisions in the web, but thought circumferential removal might be better. Splitting them blindly, as described here, seems to be sufficient. However, the actual split must be felt as a sudden giving way, the stricture must become impalpable from without, and neither the finger from below nor the No. 30 (English) bougie from above should be gripped afterwards. Mere stretching is not enough. They may be stretched enough, without splitting, to allow the passage of the bougie and the finger, but in that case the bougie and finger will be gripped firmly as they pass.

I believe that these annular strictures are merely one variant of fibrous stricture due to peptic oesophagitis and that they are, therefore, not congenital. One of the two cases reported here occurred in a woman aged 47 who had had dysphagia for one year, and the other occurred in a woman of 84 who had had dysphagia for 15 years. Thus the first had no dysphagia till the age of 46 and the second till the age of 69 . The symptoms of reflux oesophagitis due to the associated hiatal hernia preceded the dysphagia by a few months in the first and by about 20 years in the second. On their histories, it would be absurd to regard either as congenital. I do not know why peptic oesophagitis sometimes results in strictures of this annular type with the fibrosis confined mainly to the mucosa.
In two other cases with a low stricture the hiatal hernia and stricture were first noticed after lower subtotal gastrectomy for chronic duodenal ulceration. In both a careful history showed that reflux oesophagitis and dysphagia had begun before the gastrectomy.

The first was a woman, aged 59, who had a gallbladder full of stones removed three years before without relieving her indigestion. At this operation, scarring of the first part of the duodenum was noticed. During the following two and a half years she had six severe haematemeses, two requiring blood transfusions, all assumed to come from a duodenal ulcer. For three months the pain, previously confined to the epigastrium, spread up behind the sternum and was associated with regurgitation of food, which was regarded as vomiting, and loss of weight. On November 3, 1959, without any previous barium examination, a Polya gastrectomy was done. No comment on the hiatus was made. A Ryle's tube was left for a few days down the oesophagus into the stomach remnant for post-operative aspirations in the usual way. As soon as it was removed and she began to take fluid and soft food by mouth the retrosternal pain and vomiting returned exactly as it had been for the preceding three months, but it rapidly became worse. It was then noticed for the first time that the "vomiting" was really regurgitation from the oesophagus, and a thoracic surgeon was consulted. Her first barium examination was ordered and showed complete obstruction in the lower oesophagus. Repetition of this examination after oesophagoscopic dilatation of the very tough stricture showed a large hiatal hernia. The operation described here was done on January 6, 1960, and she is now well with no pain and no dysphagia.

The second was a man aged 64 who began to have pain characteristic of duodenal ulcer at the beginning of 1959 and perforated his ulcer on May 24, 1959. It was oversewn. From then on he had typical symptoms of reflux oesophagitis, and early in September, 1959, food began to be regurgitated and he rapidly lost weight. On September 30 a massive haematemesis required a transfusion of 5 pints of blood followed by an emergency laparotomy at which a Polya gastrectomy was done and the duodenal ulcer, which was not bleeding, was left in situ. The hiatus was not mentioned. Dysphagia and regurgitation of food continued, but no notice was taken until his weight was reduced to 5 st. (normal 10 st.) and he required intravenous fluids. $\mathrm{He}$ was then referred to the thoracic surgeon, and a barium examination (his first) showed complete lower oesophageal obstruction (Fig. 4). At oesophagoscopy easily dilatable narrowing was found from 31 to $36 \mathrm{~cm}$., but at $36 \mathrm{~cm}$. there was a very tight, fibrous stricture which was dilated with difficulty using more force than usual in the hope of enabling him to swallow for a period during which his general condition could be improved preparatory to further surgery. This hope was not realized. A 
few hours later he had severe abdominal pain and rigidity, and a radiograph showed gas under the diaphragm. He was returned to the theatre, opened through a left thoraco-abdominal incision and the perforation by a bougie found through the oesophagus just below the phreno-oesophageal ligament into the peritoneal sac of a small hiatal hernia. Oesophagoplasty and repair of the hernia were done. When the phreno-oesophageal ligament was sewn to the under surface of the hiatus the oesophagoplasty was entirely below the diaphragm. He died suddenly on the fifth day, probably of a coronary occlusion. The oesophagoplasty was intact. (This is the patient who had the enormous hypertrophy of the oesophageal muscle.)

These two cases correspond to those described by Bingham (1958) and McCredie and McDowell (1958) in which oesophageal strictures became evident after subtotal gastrectomy for duodenal ulcer. McCredie and McDowell's attention was focused on the possible part played by the stomach tube in causing the stricture, and they suggested that perhaps rubber stomach tubes should be avoided. Bingham realized that the main cause is the missed hiatal hernia which was subsequently found in all the cases, but he did not suggest that the way to avoid the calamity is to investigate the patient properly before the gastric operation. Then the presence of the hiatal hernia would be known and it would be repaired at the same time. Cases such as this show that lower subtotal gastrectomy for hiatal hernia with stricture is not always as successful as MacLean and Wangensteen's (1956) experience suggested. It may make the oesophagitis and stricture worse, possibly due to reflux of bile as stated by Wells and Johnston (1955).

The operation, as described (freeing the oesophagus, splitting the stricture internally, and repairing the hernia), was done on eight patients through a left thoracotomy. Of these, six had low short strictures (two of them annular), one had a high short stricture, and one a long stricture. In five, bouginage from above sufficed to split the stricture. Retrograde dilatation through a gastrotomy was necessary in the other three.

One patient had a penetrating ulcer near the stricture which had leaked into the mediastinum, making a narrow, fibrous track lined by granulation tissue (Fig. 5). This track was dissected to its origin from the oesophagus, cut off level with the submucosa and the small opening closed with a catgut stitch and covered by closing the oesophageal muscle over it with two linen stitches. Otherwise the operation proceeded as described, with retrograde internal splitting through a gastrotomy. Another patient shared with my colleague, Mr. E. E. Dunlop, had a high, short stricture which was split by bouginage through the oesophagoscope followed by repair of the hiatal hernia through the abdominal route under the same anaesthetic. Four years before, dilatation of the stricture by another surgeon, without repair of the hernia, had produced only temporary relief of dysphagia.

The above 10 patients are now all well with no dysphagia and need no dilatations, but six of them required a period of post-operative selfbouginage, and one, an irascible old man who flatly refused to swallow the bougie, needed one post-operative oesophagoscopic dilatation.

One patient with a chronic duodenal ulcer and hiatal hernia was referred to me for endoscopy only. I found a tight low stricture which I was only able to dilate to size No. 22 (English). He was the patient of a general surgical colleague whom I advised to do a Polya gastrectomy, retrograde splitting of the stricture, and repair of the hernia through a left abdomino-thoracic approach. $\mathrm{He}$ did the gastrectomy and repaired the hernia, as advised, but omitted to split the stricture. $\mathrm{He}$ simply passed the size 22 bougie again. The patient's indigestion and pain were relieved, but he was still unable to swallow solids when he was referred back to me five months later. Oesophagoscopic dilatation was then successful up to size No. 28. Presumably the five months' freedom from reflux made it easier. A subsequent period of a few months of self-bouginage with a size No. 26 Hurst bougie prevented return of the dysphagia.

Two patients had an oesophagoplasty. One, whose oesophagus was perforated with a bougie during endoscopic dilatation, has already been described. His death was the only one in the series. The other was the one with the high stricture already mentioned in which the fibrosis seemed to involve the whole oesophageal wall. The chest was opened through a right thoracotomy, but the stricture was so firmly attached to the membranous part of the lower trachea with no plane of cleavage that I was afraid of opening the trachea and foolishly abandoned the dissection halfway. I made a small vertical oesophagotomy below the stricture where the whole wall, including the mucosa, appeared macroscopically normal. Through this, I began retrograde dilatation with Hegar's dilators. The stricture was about $2 \mathrm{~cm}$. long and the lumen hard to find. Finally a small dilator found it, but, when pushed, it emerged through the oesophageal wall half-way up the stricture. This would probably not have occurred if the stricture had been completely freed so that the dilator could be guided by encircling fingers. The abandoned dissection then had to be completed (the trachea was not opened) and an 
oesophagoplasty done. He required one postoperative oesophagoscopic dilatation followed by self-bouginage.

The last of the 14 cases did not have the operation described because he was a cardiorespiratory cripple with hypertension and gross emphysema who could barely walk 20 yards. He had oesophagoscopic dilatation at intervals of about six months until the stricture became impassable. The dilatations were never satisfactory because he used to become more and more cyanosed under anaesthesia and I had to stop when ordered to by the anaesthetist. Then, since it was the only operation he was thought able to survive, he had a gastrotomy under local anaesthesia on March 29, 1957. He still lives, now aged 70, in bed and in his wheeled chair. Periods occur when fluids will pass the stricture and then he takes less by the gastrotomy tube. $\mathrm{He}$ is included in this series because he was treated during the same period and because his story shows that dilatations without repair of the hernia are not of lasting benefit.

\section{Discussion}

Surgical treatment should always have a sound pathological basis. The above treatment of this type of case is based on the following beliefs about the underlying pathology, which, I realize, are different from any hitherto put forward and are as yet unproved, but which I nevertheless consider to be close to the truth, because the treatment based on them is usually effective and they seem to be in accord with the pathological findings and with general pathological principles.

Most cases of reflux oesophagitis show hyperaemic mucosa with shallow superficial ulceration and sloughs which may come and go for years, with some spasm and dysphagia at times, but no fibrous stricture. Even at this superficial stage there may be oedema and enlarged lymph nodes in the surrounding mediastinum. In periods of quiescence some healing occurs. The squamous epithelium may grow again in ulcerated areas and may develop leucoplakia. In other cases, or at other times in the same case, the denuded areas may be replaced by metaplastic junctional epithelium (Hayward, 1961b). This gives some protection from acid-peptic digestion, and future inflammation will be more marked in the area immediately above still lined by squamous epithelium. With continuation or repetition of the process of superficial ulceration and healing in cases characterized by replacement by metaplastic junctional epithelium, this columnar epithelium creeps higher and higher. It may reach the level of the arch of the aorta.

In a few cases, probably as a rule quite suddenly, and for reasons still unknown, the previously very superficial inflammation undergoes violent transmural spread. This seems always to occur in the region where the squamo-columnar junction happens to be at the time and may therefore be anywhere from about $2 \mathrm{~cm}$. up the oesophagus to the level of the arch of the aorta. The transmural spread is circumferential and symmetrical. Within weeks there is a circle of contracting fibrous tissue in the mucosa and submucosa and usually severe peri-oesophagitis outside with rigid fibrous adherence of the gullet to all surrounding structures at this level. The muscle coat in between is astonishingly little affected. It is scarcely ever destroyed like the tissues of the mucosa and submucosa. On the contrary it is often hypertrophied. There may be some oedema and a little increase in fibrous tissue between the muscle fibres.

This evenly circumferential transmural inflammation is quite different from the transmural spread in one place of a penetrating ulcer with destruction of all coats, though rarely a penetrating ulcer may also be present in the vicinity (Fig. 5). Usually the associated ulceration is still quite superficial. It may also be circumferential but is more often confined to one part of the circumference. As the severity of digestion by regurgitated gastric contents abates the superficial ulceration may heal more or less completely, but the fibrous tissue in the mucosa and submucosa remains tough and contracted so long as any reflux is occurring. The site of the stricture is therefore in the mucosal and submucosal layers, not the muscular layer. It is the intact muscle layer which makes internal splitting, as described, feasible. When inflammation spreads up above the stricture-so-called ascending oesophagitis-it usually remains superficial, and the narrowing which extends slowly upwards is due mainly to muscular hypertrophy and spasm. The fibrous stricture which requires internal splitting is usually still quite short and at the bottom of the narrowed area.

There is a widespread delusion that abnormal tough fibrous tissue is of necessity permanent. Once the adjectives "fibrous," "organic," or " chronic" are applied to a stricture, most surgeons automatically consider that it probably ought to be cut out. This idea is false, and the operation described here is based on the opposite view that abnormal fibrous tissue anywhere in the body, no matter how inert the fibrocytes and bundles of collagen may appear under the microscope, is 
really a labile tissue which only persists if there is a cause continually acting to keep it there. For instance, pleural adhesions often disappear, a very thickened pleura may return to normal if the cause of the inflammation has been completely eradicated, and the thickest keloid may in time be reduced to paper thinness.

It so happens that in the type of fibrous oesophageal stricture under discussion the cause, namely oesophageal reflux, can be completely stopped (by efficient repair of the associated hiatal hernia). With the cause removed the ulcers can re-epithelialize and the fibrous tissue under them thin out like a keloid. This can occur even without the complete internal splitting advised here, as was shown by MacLean and Wangensteen (1956). Restoration of the normal mucosal and submucosal architecture where it has been destroyed is, of course, not to be expected, but the gullet can function again as an efficient conductor of food to the stomach.

The operation is also based on the belief that these strictures are all acquired and all due to oesophageal reflux, which is in turn almost always, if not always, due to an associated hiatal hernia. This is at variance with other opinions. For instance, Berman and Berman (1959) state that "the diaphragmatic defect may well be of ancillary importance." MacLean and Wangensteen (1956) reported 18 cases of fibrous stricture in which, according to them, only eight had a hiatal hernia, and they only considered it worth while to repair the hernia in three of these eight. Sweet et al. (1954) reported 50 cases of which 48 had hernias. Though all were treated surgically in various ways they do not mention repairing the hernia in any of them. All my cases have had a hernia, though in three it was quite small. The discrepancies in the reported incidence of hiatal hernia with these strictures seem only to be explicable by assuming that the hernia has often been missed. The fact that oesophageal dilatation alone gives only temporary relief of dysphagia, whereas repair of the hernia as well and no other treatment, medical or surgical, has in my cases always been followed by prolonged relief, is evidence which suggests that the hernias really were present and caused the strictures and were responsible for their persistence.

As well as these pathological considerations, some fundamental surgical principles are involved. Surely it is a surgical principle that a damaged organ which can be restored to normal function should not be removed. These strictures fall into this category and this should be reason enough for not resecting them. When, moreover, their resection makes it necessary to cut out a piece of $\frac{0}{0}$ jejunum or colon to bridge the gap the situation 등 is comparable with a woman cutting up new $\frac{\bar{\sigma}}{}$ clothes to patch old ones. This sort of thing is justifiable when the stricture is malignant but hardly when it is benign and reversible.

Still worse is the practice of attacking perfectly $\overrightarrow{0}$ normal stomach, cutting it this way and that and $\vec{\overrightarrow{ }}$ removing more or less of it here and there, or $\vec{\sigma}$ wrecking its nerve supply and its pyloric sphincter, $\overrightarrow{\vec{x}}$ to deal with a condition which involves the $\vec{x}$ oesophageal hiatus and the oesophagus. This, of $\vec{\sigma}$ course, followed the discovery that oesophago- $\overrightarrow{\vec{A}}$ gastrostomy with normal stomach merely increased of the reflux and caused the stricture to recur at the 음 anastomosis; so surgeons proceeded to make the stomach as abnormal as possible before the $z$ anastomosis. It is a good surgical principle, when- $\frac{\aleph}{\Delta}$ ever possible, to remove the cause of the trouble $\vec{\partial}$ and not to try to minimize its effects by partial $\vec{\mathscr{}}$ or complete destruction of the function of other $\stackrel{\circ}{-}$ normal organs. The cause in this condition is 0 acid-peptic reflux and the proper treatment is to stop the reflux, which is quite simply done by repairing the hernia, not to stop the normal function of gastric secretion which is necessary to $\frac{\circ}{D}$ good health.

Nothing can replace the satisfaction of $\overrightarrow{\overrightarrow{0}}$ savouring food in the mouth and feeling it pass freely down the gullet into a normal stomach, normally situated in the abdomen and with its vagal nerve supply intact. The treatment described here usually achieves this result.

\section{SUMMARY}

There is no agreement on the management of $\frac{\delta}{\beta}$ patients with fibrous oesophageal strictures due to peptic oesophagitis. Many widely different approaches to the problem, often involving more $\frac{D}{2}$ or less extensive resections, have been advised.

This paper is based on the experience of treating $N$ 14 personal cases.

This experience has led to the conclusion that the best method of treatment for most of these $\tilde{\omega}$ patients consists of an operation in which the steps are freeing the strictured part of the oeso- 0 phagus, splitting the stricture internally and repairing the hernia, followed by post-operative $\stackrel{?}{+}$ self-bouginage for a limited period when necessary. The hernial repair must include proper fixation $\frac{\vec{\Phi}}{\mathbb{D}}$ of the oesophageal attachment of the phreno- $\frac{\mathcal{Q}}{\Phi}$ oesophageal ligament under the hiatus.

This treatment is based on the opinion that these strictures are acquired, are due to oesophageal $\delta$ reflux and can be relaxed permanently after the 
reflux is stopped. It is thought that the reflux is almost always due to a sliding hiatal hernia which may be small and has probably often been missed.

Resections for this condition are condemned.

\section{REFERENCES}

Allison, P. R. (1951). Surg. Gynec. Obstet., 92, 419.

- Wooler, G. H., and Gunning, A. J. (1957). J. thorac. Surg., 33, 738.

Barrett, N. R. (1952). Proc. roy. Soc. Med., 45, 279.

- (1957). Surgery, 41, 881.

Belsey, R. H. R. (1953). Trans. med. Soc. Lond., 68, 18.

Benedict, E. B., and Nardi, G. L. (1957). Amer. J. Surg., 93, 238.

Berman, J. K., and Berman, E. J. (1959). A.M.A. Arch. Surg., 78, 889.

Bingham, J. A. W. (1958). Brit. med. J., $2,817$.

Bugden, W. F. (1957). Amer. J. Surg., 93, 248.

Burke, J. B. (1959). Brit. med. J., 2, 787.

Byrnes, C. K. (1958). Irish J. med. Sci., p. 65.

Cross, F. S., Smith, G. V., and Kay, E. B. (1959). J. thorac. Surg., 38, 798.

Ellis, F. H. (1956). Proc. Mayo Clin., 31, 615.
Ellis, F. H., Jr., Andersen, H. A., and Clagett, O. T. (1956). A.M.A. Arch. Surg., 73, 578.

Groves, L. K., Martinez, W. V., and Effler, D. B. (1959). J. thorac. Surg., 38, 537.

Hayward, J. (1961a). Thorax, 16, 36.

- (1961b). Ibid., 16, 41.

Holinger, P. H., Johnston, K. C., Potts, W. J., and daCunha, F. (1954). J. thorac. Surg., 28, 345.

Lindskog, G. E., and Kline, J. L. (1957). New Engl. J. Med., 257, 110.

McCredie, J. A., and McDowell, R. F. C. (1958). Brit. J. Surg., 46, 260.

MacLean, L. D., and Wangensteen, O. H. (1956). Surg. Gynec. Obstet., 103, 5

MacMahon, H. E., Schatzki, R., and Gary, J. E. (1958). New Engl. J. Med., $259,1$.

Mustard, R. A. (1957). Canad. med. Ass. J., 76, 811.

Smith, L. C., and Bradshaw, H. H. (1959). Surg. Gynec. Obstet., $109,230$.

Sweet, R. H., Robbins, L. L., Gephart, T., and Wilkins, E. W. (1954). Ann. Surg., 139, 258.

Tanner, N. C. (1955). Lancet, 2, 1050.

Wells, C., and Johnston, J. H. (1955). Ibid., 1, 937.

Wooler, G. (1956). Thorax, 11, 275. 